\title{
Semantic enhancement of forest inventories by reengineering
}

\author{
H. Hasenauerl) G. Pernul ${ }^{2)}$ \\ 1) Institute of Forest Growth and Yield, Department of Forestry, \\ University of Environmental Sciences, \\ Peter Jordan Str. 70, A-1190 Vienna, Austria. \\ E-mail: hasenau@edv1.boku.ac.at \\ 2) Institute of Applied Computer Science and Information Systems, \\ University of Vienna, Liebigg. 4/3-4, A-1010 Vienna, Austria. \\ E-mail:pernul@ifs.univie.ac.at
}

\begin{abstract}
In order to enhance the semantic representation of data collected in forest inventories the paper proposes a reengineering technique consisting of Entity-Relationship modelling and a transformation process into relational database structures. The work is motivated by the fact that a huge amount of information about our most complex ecosystem on earth, the forest, is available but unfortunately currently only interpretable by the forest experts. This is due to the current structure of the data and in our opinion this is a huge disadvantage because data collected may also be of tremendous value in related disciplines, for example, for solving ecological questions. Enhancing the semantics and providing a more convenient user interface for accessing the data may make the information available in a much easier way to the ecologist, the botanist, the wildlife expert, and to all other parties that might be interested in using the inventory.
\end{abstract}

\section{Keywords}

Data modelling, reverse database engineering, forest inventories, semantic enrichment

\section{INTRODUCTION}

Forest inventories are carried out by forest companies for collecting information about their forest lands in order to develop efficient forest management plans. Another type of forest inventories are implemented by the national forest experimental stations of European countries (for example, in Austria, Finland, or Germany) with the main purpose to estimate and control the forest covered land areas of their countries and to observe the state of forests under changing environmental conditions. 
Today most forest inventories are computerized but are mainly designed and used by forest experts and the data collected are generally coded and stored as simple ASCII files. For extracting information several application programs must be developed and applied to the data.We will refer to such a file structure as flat files in the following.

While for certain forestry applications (and also for the forest engineers) the structure of the data has proven over the recent years to be sufficient newer applications, for example, software support for environmental protection, ecosystem software, or ecological management software can only make limited use of the data because of its current structure. This is a huge disadvantage because the data collected may be of tremendous value for solving ecological questions. This is because forests are considered as one of the most complex and highly developed ecosystems and thus react to changing environmental conditions rapidly. From the viewpoint of an ecologist, who unfortunately rarely is also an expert in forestry, reality may be seen differently from what was considered by the forest engineer at the time the structure of the forest inventory was determined. It might be a realistic but the worst case that it is the opinion of the ecologist that data has a poorly designed structure, applications are poorly coded, use poor logic, are poorly documented and for him as a consequence the whole forest inventory does appear as not representing the semantics of the application domain properly. This situation is a very good example of the fact that it is not possible to get a system permanently complete, because it is not possible to predict now what we will want the system to do ten, twenty, or even thirty years from now.

As a solution to the problem discussed above we suggest to reengineer existing forest inventories in order to make information about our forest available and better interpretable for non forest experts. Reengineering is a research discipline within Software Engineering with its main purpose to carefully analyze a given system, to identify the system's components and their interrelationships, to create another - more advanced - form of the system at a higher level of abstraction (i.e. to reverse engineer the system), and finally to use the abstractions to reconstitute the system in a new semantically enriched form. As a result the new version of the system should be able to better meet the new requirements in comparison with the old form of the system.

The objective of this paper is twofold: 1) We develope a robust methodology to reengineer information stored as flat file structures in existing systems. The methodology uses state-ofthe-art data modelling and database design concepts in order to enhance the semantics of the new form of the system and to reduce redundancy, inconsistencies, and system maintenance costs. 2) We report our experiences in using this methodology in a case study on the reengineering of one of the most complex forest inventories in Europe - the Austrian National Forest Inventory, OEFI (1994). A prototype application has been developed in MS Access to test the adequacy of the proposed methodology.

The outline of this paper is as follows: In the remainder of Section 1 we discuss related work. In Section 2 we describe the current form of the forest inventory used as the starting point of our efforts. In Section 3 we explain the methodology developed to reengineer flat file structures. For its understanding some basic knowledge of data modelling and the relational data model is necessary. Section 4 is devoted to the case study. In Section 5 we conclude our work and discuss open research questions.

\section{Related Work}

Reverse software engineering is a recognized research topic in which a huge amount of work has been published recently. Instead of referring to particular articles we find it more useful to point only to three special issues of prestigious periodicals devoted to reverse software engineering, the CACM (1994), Software (1990), Software (1994). Articles appearing in these special issues can be consulted to gather further references. Our work is also related to conceptual and logical database design. For conceptual database design the EntityRelationship (ER) Model of Chen (1976) and its variants serve as a defacto standard and were also chosen as the underlying conceptual data model for reverse engineering in our approach. Database design in general is treated in detail, for example, by Navathe and Pernul (1992), by 
Batini et. al (1992), or by Theorey et. al (1986). Although considered as an important topic methodologies for reengineering of databases are not reported in the literature frequently. Navathe and Awong (1987) propose a systematic approach for the reverse engineering of relational databases into extended ER models, Markowitz et. al $(1989,1990)$ focus on the more theoretical aspects of reverse database engineering and Premerlani and Blaha (1994) use an object-oriented design and analysis for the reverse engineering of relational databases. We are not aware of any other project devoted to the reverse engineering of forest inventories.

\section{THE APPLICATION ENVIRONMENT}

The application environment used in this study is given by the design and data collecting concept of the Austrian National Forest Inventory. The tasks and the data collecting system as it is shortly described below is well documented in a manual called "Instructions for the field work of the Austrian Forest Inventory", OEFI (1994).

The general objective of the OEFI is the recording of the Austrian forest covered land, the timber growing stock, the increment and the felling quantity. In the sixties the sample plot system was introduced and currently the OEFI consists of about 10.000 permanent sample plots and every year a fifth (five year measurement cycle) of the plots spread all over Austria are remeasured. During the last decade the tasks have been extended rapidly and today a huge amount of data are recorded on a plot and tree basis for describing site and stand characteristics, stand structure and treatments, regeneration, damages by game, and which is of increasing interest - variables for characterizing the health conditions of trees and stands.

Because a permanent plot system was installed always the same plot location and even the same trees on a plot are observed. The advantage of such a system is that the current status can be compared with previously ones and so the change itself can be considered directly.

The data collected can distinguished into plot and single tree related variables. The plot related information is collected on a fixed plot size of $300 \mathrm{~m}^{2}$. Related variable groups describe some general plot information, like the percentage of forest covered land, land owner, political district etc., common stand data, like stand structure, age group, growth class, growth district, etc. Furthermore, a very detailed information is given about the site conditions of the plot by considering the soil and humus conditions, the steepness, the elevation, the exposition, etc.

The recording of single tree related information is dependent on two different plot size: For trees with a diameter at breast height $(\mathrm{DBH})$ of more than $10,4 \mathrm{~cm}$ the angle count system with a basal area factor of four is used for selecting the sample plot belonging trees. The angle count system gives thicker trees a higher probability to be included in the sample than thinner ones because they contribute a higher percentage of the basal area or volume per unit area and should therefore be measured more accurately. From the statistical point of view it is important to note that every tree has its own sample plot size depending of its DBH. For trees with a DBH between 5,0-10,4 cm again a fixed plot size of $21 \mathrm{~m}^{2}$ is used. Trees with a DBH of less than $5 \mathrm{~cm}$ are not included.

For every tree the location on the plot is defined by measuring the azimut and the distance between the tree and the permanently marked plot center. Tree species, $\mathrm{DBH}$, total tree height, height to the base of the live crown, etc. are determined. Furthermore the tree class for characterizing the tree's competition situation and some variables describing the size of tree damages like stem and root damages, number of bark peelings, the crown condition and the needle fall are given.

Finally some general economical information concerning the expected wood quality, the cutting and logging conditions, like distance to the next road etc. and wildlife and game management variables are points of interest as well.

The general file structure of the forest inventory is a flat file consisting of ASCII coded values. In the example shown in Figure 2 (which is already a simplification of the original data set for a given sample plot) the total number of lines to be used can be identified by the first 
two digits. For example, the first sample plot has a total of 25 lines. The first five are common plot information collected on the large fixed plot area of $300 \mathrm{~m}^{2}$. The remaining lines indicate the number of recorded trees on the plot with their observed variables values.

It is obvious that such a system requires a great knowledge of the data itself and it is almost impossible to keep all the different variables in mind. The current situation of interacting with the system requires a forestry expert who, for many interpretations of the data, needs to be consulted. On the other hand researchers of other disciplines have got an increased interest to investigate forest areas because they represent our highest developed ecosystem and it is assumed that under changing environmental conditions (global warming, etc.) forest areas will respond very quickly and sensitively. This is because forest is considered as one of the key indicators for detecting and estimating environmental caused changes. It is important to note that one of the most complicated parts in quantifying the external impacts on our ecosystem is to distinguish between natural stress factors as they result from growing competition within a stand and the external impact caused by air pollution itself.

It can be summarized that we have a very well designed example for a forest inventory with a lot of information about one of our most complex ecosystems. On the other hand we have non forestry experts (ecologists, wildlife people, botanists, etc.) who have an increased interest in our forest data but access of the existing information is hardly possible for them. Another important reason which also suggests the urgent need of an adequate database reengineering is that the collection of environmental data is very expensive and whenever researcher have the chance to use already existing information a lot of money can be saved.

\section{REENGINEERING PROCESS}

For reengineering flat file structures we propose an approach consisting of four main building blocks and several major design activities. Two of the blocks are again decomposed into further development steps. Figure 1 gives an overall picture of the methodology in terms of life-cycle phases.

Building_block_1: Existing System

The first building block is the existing system and the activity to be performed is analysis and redocumentation. Redocumentation is focused on understanding the so-called mission of the existing system. The outcome of this phase is a set of requirements specifications, which are usually rather informal, disorganized, and expressed in natural language at this step of design. These specifications are used as a basis for reverse engineering of the existing system.

Building_block_2: Entity-Relationship Model

Reverse engineering is understood as the process of analyzing the existing system in order to identify its components and their interrelationships and to create a representation of the system in a high level of abstraction. The ER model is used for this purpose. This phase first consists of determining the entity types, their attributes and corresponding domains, as well as the integrity properties that must hold. When the structure of the entity types is determined candidate keys and foreign key groups must be determined. Candidate keys are used to uniquely identify each entity and foreign key groups help in determining relationships between entity types or to find generalizations. The development of an ER model is an iterative process, often involving trial and error. We refer to this process as restructuring. It is essential that the designer gets an exact understanding of how the different components of the ER model interact and what exactly their meaning is. In order to develop a proper solution experienced designers as well as a close interaction with application domain experts is necessary during this phase.

Building_block_3: Experimental prototype

This block is in close relationship with block 2 and consists of the development of an experimental prototype of the application in order to verify the design. With real examples 
from the application domain potential users of the system may test its appropriateness and design errors at the conceptual level can already be found at this early step of design. This phase is performed by classical forward engineering and stepwise refinement. With forward engineering the process of moving from high-level abstractions and logical implementationindependent specifications (i.e. the ER model) to the physical implementation (the experimental prototype) is meant while stepwise refinement refers to the iteration process of refining the prototype based on corrections at the high-level specifications and vice versa. This process must be continued until the prototype implementation completely meets the users requirements.

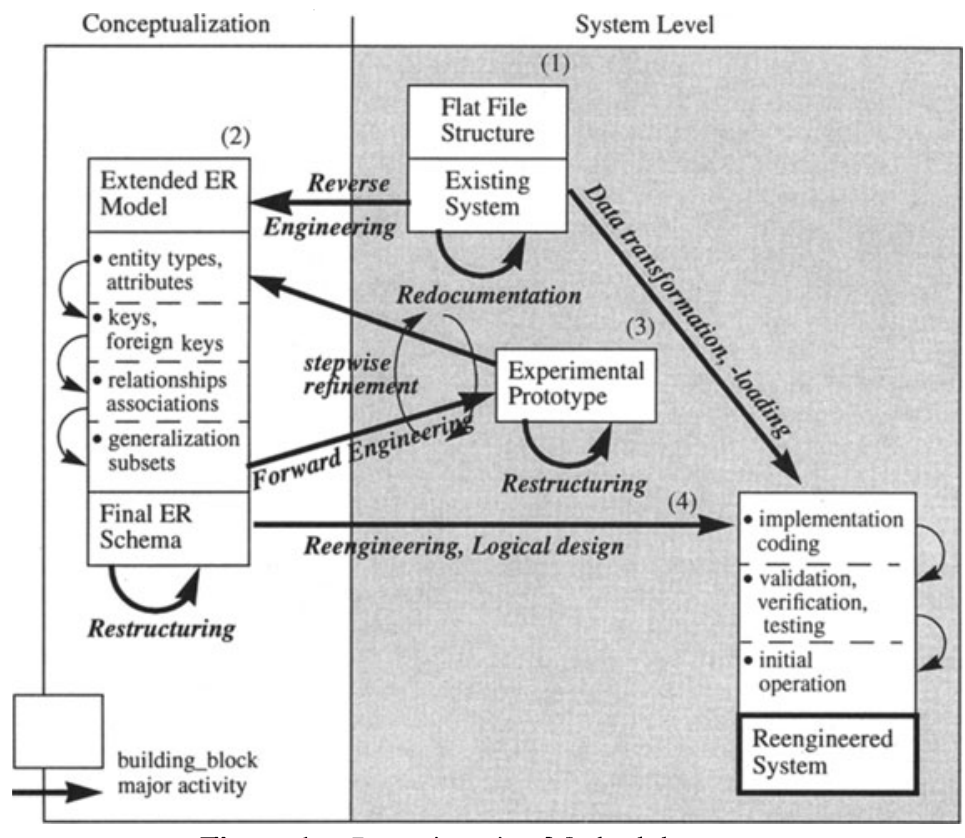

Figure 1 Reengineering Methodology

Building_block_4: Logical design, reengineered system

While the earlier phases of design are more intuitive and rely on the experience of the designer for this block it is possible to give some more exact rules how the new form of the system may be determined. Input to logical design is the final conceptualization of the application. Logical design consists of the transformation of the system independent high-level abstraction from block 2 into the representation of the data model of the reengineered form of the system, in our case in the relational data model. In the following we look at each concept of the ER model in detail to see how it can be transformed into concepts supported by the relational model:

\section{Entity Types, Weak Entity Types and Multivalued Attributes}

For each entity type E of the ER schema, we create a relation schema RS which includes all attributes that can have atomic attribute values only. By analyzing the functional dependencies valid in RS, the set of candidate keys may be derived, and one candidate key is chosen as a primary key of RS. Foreign key attributes or relationship attributes are not included yet; they will be added during subsequent steps.

For each weak entity type (WE) (i.e. entity type which may not exist without associated 
strong entity type) associated to E (strong entity type), we need to create an additional RS and include the attributes with atomic domain of WE in RS. In addition, we include the primary key attribute of the relation that resulted from the corresponding strong entity type $E$ as a foreign key into RS. The primary key of the relation schema that resulted from WE is the combination of this foreign key and the partial key of the weak entity type.

For each multivalued or composite attribute (i.e. attribute having a non-atomic domain) $\mathrm{A}$ in $\mathrm{E}$ we create a new RS that includes an entity set $\mathrm{X}$ corresponding to A plus the primary key $\mathrm{K}$ of $\mathrm{E}$.

\section{Relationship Types}

To transfer relationship types into a relational database schema we have to distinguish between $\langle 1: 1\rangle,\langle 1: \mathrm{N}\rangle$, and $\langle\mathrm{N}: \mathrm{M}\rangle$ cardinalities as well as between mandatory and optional participation of the corresponding entity types in the relationship type.

In general, two entity types E1 and E2 participating in a $<1: 1>$ relationship type result into two separate relation schemas. However, in the case of total (mandatory) participation of E1 and E2 we can also consider the possibility of merging them into a single RS.

For each binary relationship type of cardinality $<1: \mathrm{N}\rangle$ we first have to identify the entity type $\mathrm{E} 1$ on the 'many' side. If the participation is mandatory, we include the primary key of relation schema RS2 that represents the entity type at the other side of the relationship type as a foreign key in RS1. If the participation of the entity type participating on the 'many' side is optional, we can also consider to set up three relation schemas; one for each participating entity type and one for the relationship type.

For each relationship type of cardinality $<\mathrm{N}: \mathrm{M}>$ between two entity types E1 and E2 we develop an additional relation schema RS and include both primary keys from E1 and E2 as attributes. These attributes also form the primary key in RS.

\section{Generalization, subset hierarchies}

A generalization hierarchy of the ER model is transformed into a relation schema RS1 for the generic entity type and a separate relation schema RS2, ..., RSn for each of the specialization types. RS1 contains the generic entity type key and all common attributes including the common attribute on which the decomposition is based. Each specialization relation (RS2, ..., $\mathrm{RSn}$ ) contains the generic entity type key as well as all attributes specific to the subsets. There is no difference in generalization and subset hierarchy in mapping into the relational data model, except interrelation integrity properties.

Logical design results in relational schemas which can be implemented by using the data definition language of a relational database management system (DBMS). In building_block_4 implementation and coding is concerned with the programming of the final operational version of the reengineered database application, the definition of the database schemas and views, the specification of the users and user groups and the definition of authorization and access control structures. Validation, verification, and testing are aimed at assuring that each previous phase of development is of acceptable quality and is an accurate transformation from the previous phase. The operational phase includes the actual data loading and an initial operation phase may be necessary to correct errors that were not detected in earlier phases. For data loading transformation programs must be provided that map the data of the flat files of the initial system into the representation of the reengineered system.

\section{CASE STUDY}

Based on an existing file structure a first ER diagram for the forest inventory has been reengineered. In this section we draw our attention on a specific subset of the data available from which we believe it might have potential for solving ecological questions. This subset is concerned with tree damages and related information. 
In Figure 2 a general overview of the redesign process with respect to 'damages' is given. The process starts with identifying relevant information in the flat files, continues with developing an ER model (this step includes close interaction with the domain experts) and concludes with transforming the ER into relational data structures. The redesign is driven by the following motivation: We distinguish two different kinds of possible forms of damages: biotic and abiotic. Biotic are all damages caused by living individuals like human beings, animals, insects, viruses or wood destroying fungus. With abiotic we refer to all external environmental impacts to the ecosystem forest like wind, snow, frost, heat, etc. and all kind of damages caused by them. Abiotic and biotic damages are disjoint, i. e. a particular damage may only be assigned to one of the groups. However, both classes of damages have also properties in common. In particular, these may be sample point information, tree related information, each damage has a unique code, is in a certain development phase, makes bio/ chem control necessary, the tree has a certain chance to recover from it, and so on. These common attributes indicate, that a generic entity type may be a proper solution to represent damages in a database and because of the disjointness condition of the specializations, modelling a generalization structure may be adequate. As can be seen in Figure 2 the distinction into biotic and abiotic damages is based on attribute 'type' and both specializations have different additional damage relevant attributes. For abiotic damages high-diameter ratio, rem. crown length, crown class while for biotic damages animal, part, gallery are relevant.

In a further hierarchy we decided to represent in the ER diagram the fact that we have different types of damages caused by biotic reasons. To distinguish between them we take the fact that the damage appears on a certain part of the tree. The idea is that damages caused by insects, animals, etc. normally are apparent on typical parts of the tree because of the nutrition preferences and living rhythm of the organism. From the view of a botanist, entomologist, wildlife manager, etc. the damage affected tree part is important for identifying the species, the importance of the damage and its influence to the ecosystem, the chance to recover and its relevance for further diseases. For example if a tree is bark peeled by deer the real problem is not the peeling itself but the remaining wound is often the entering point for insects and decays which influences the future vigor of a tree much more than the peeling itself.

Considering the subsets we divided a tree into root, leaves or needles (depending on the tree species), the bark, the crown and the stem. Because an ecological system is never stabile it is a matter of fact that some tree growth affecting diseases or damages may be located on several tree parts depending on the development status of the disease. Obviously, allocating the damage into a single class is sometimes not possible. This is the reason we decided to model specialization entity types as subsets with the effect that the assignment of a certain biotic damage to several specializations is possible. From an ecological point of view this option seems to be very important because organism often extend their living environment because of the lack of nutrition in the phase of the aggregation.

When the structure of the ER model is determined, the final step consists of translating the ER model into relational database schemas. In Figure 2 this step is only indicated by showing a single table 'damage' resulted from entity type 'damage' with some sample values. A complete transformation will include a single table for each of the entity types of the ER model.

Two further things are important to note: First, the ER model as given in Figure 2 represents only an extract of the universe of discourse because relationships to other concepts like tree information, plot information, soil types, etc. are not explicitly given. Second, the ER is developed on an individual tree basis and thus is reflecting the structure of the forest inventory. With this we mean that an entity of type damage refers to a certain damage a particular instance of tree suffers from. This has the effect, that in a relational database a particular entry in a table 'damage' is uniquely determined by combining the sample plot identification with the tree identification (within the plot) and with damage identification. Including the damage code into the key is necessary because a single tree may suffer from several damages (as it is the case with tree 21 on plot 8039 in the example given in Figure 2). 


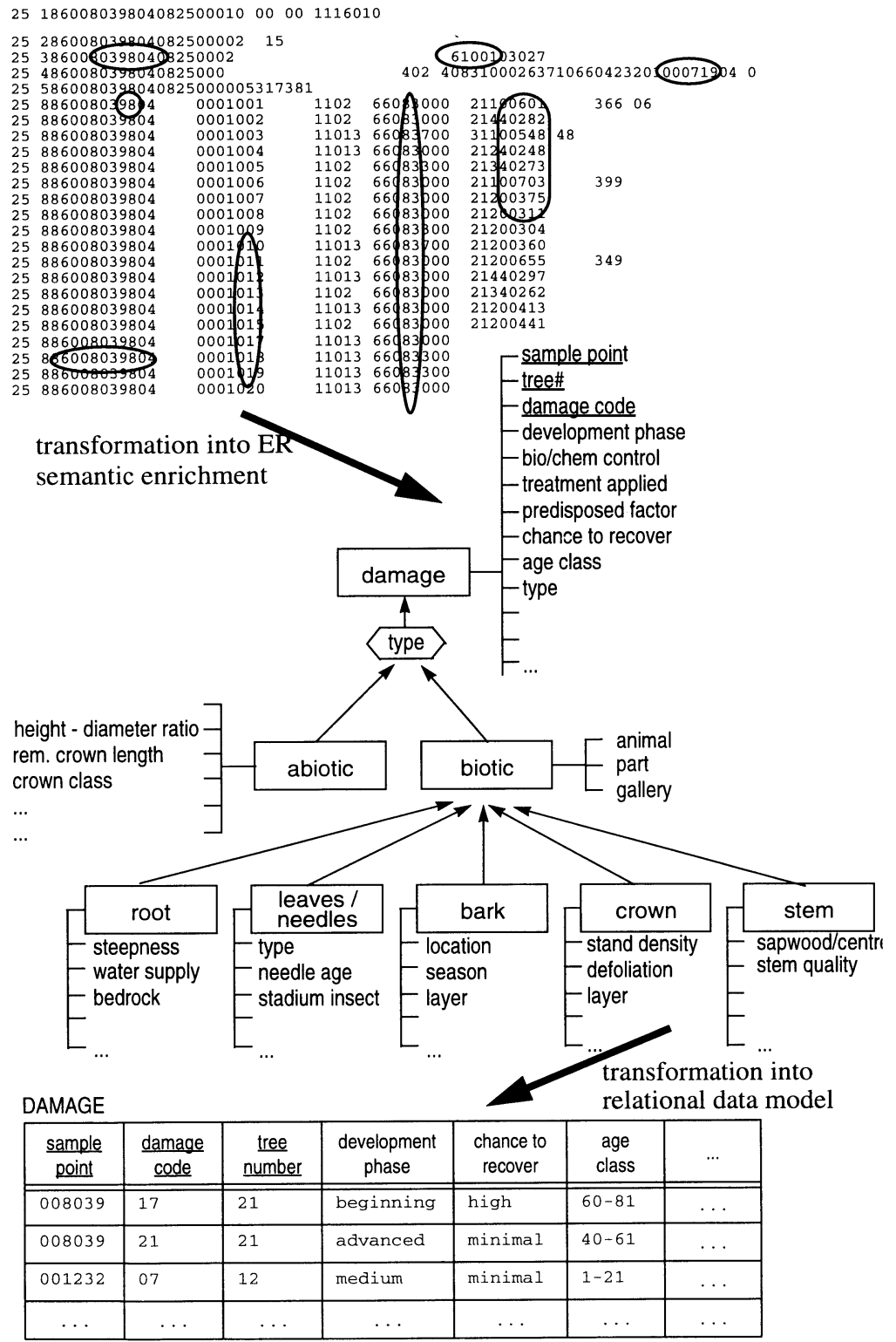

Figure 2 Case Study 
In order to enhance the semantics of flat file structured systems we have proposed a database reengineering technique. The technique uses Entity-Relationship modelling combined with a transformation process into relational databases. Resulting reengineered databases represent the semantics of the real world in a more advanced form which has the advantage that data becomes much easier accessible for non domain experts. The feasibility of performing a reengineering by using the proposed technique has been shown by a first analysis of one of the most complex forest inventories available and by transferring it into an reengineered experimental prototype system. It is important to note that our system represents a first prototype version of an ongoing project only and has not yet been proven as working properly by a large number of users.

The analysis described in this paper does only consider the static properties of a system. What is of interest is also to include functional and dynamic processes within the reverse engineering. This has turned out to be of particular importance in performing the case study. To be more precise, biotic damages/diseases and their development can be understood as a dynamic system. Starting from latent conditions, which normally do not yet effect the system negatively and often have a positive impact as a nutrition base for the useful animals the meaning of the disease can change rapidly and as a worst consequence it can even result into devastated forest areas. From a system modelling point of view it is interesting to look into modelling the conditions that are responsible for the change of the state of a disease in terms of object states and pre- and post conditions. Additional work has been devoted to this aspect in which data flow diagraming is used to model the functional part of a system while object behavior diagrams to capture the objects dynamics. First results of our efforts are reported in Pernul and Hasenauer (1995).

\section{Acknowledgment}

We would like to thank our students I. Haslik, G. Hentschel, G. Retter, R. Rosenthaler, M. Rupp, and M. Scheschy-Lun for performing part of the analysis of the forestry inventory and for producing the prototype implementation.

\section{REFERENCES}

Batini, C., Ceri, S., Navathe, S. B. (1992). Conceptual Database Design. An EntityRelationship Approach. The Benjamin/Cummings Publishing Company, Redwood City, CA, 1992.

CACM (1994). Reverse Engineering. Special Issue of Comm. of the ACM, Vol. 37, No. 5, May 1994.

Chen, P. P. (1976). The Entity Relationship Model: Towards a Unified View of Data. ACM Trans. on Database Systems (TODS), Vol. 1(1).

Markowitz, V. M., Soshani, A. (1989). On the correctness of representing extended ER structures in the relational model. Proc. of the ACM SIGMOD Conf.

Markowitz, V. M., Makovsky, J. A. (1990). Identifying extended ER object structures in relational schemas. IEEE Trans. on Softw. Eng., Vol. 16, No. 8. Aug. 1990.

Navathe, S. B., Awong, A. M. (1987). Abstracting relational and hierarchical data with a semantic data model. Proc. of 6th Conf. on the Entity-Relationship Approach.

Navathe, S. B., Pernul, G. (1992). Conceptual and Logical Design of Relational Databases. In: 'Advances in Computers', Vol 35, M. C. Yovits, (Ed.), Academic Press 1992, pp. 1-80.

OEFI (1994). Forstliche Bundesversuchsanstalt, Instruktionen für die Feldarbeit der Österreichischen Waldinventur 1992 - 1996. Fassung 1994. Eigenverlag der FBVA.

Pernul, G., Hasenauer, H. (1995). Combining reverse with classical forward database engineering - an object-oriented approch to solve the legacy system dilemma. Proc. 6th 
Int. Conference on Database and Expert Systems Applications (DEXA'95). London, UK, Sept. 1995. Springer Verlag (LNCS), 1995.

Premerlani, W. J., Blaha, M. R. (1994). An Approach for Reverse Engineering of Relational Databases. In CACM (1994).

Software (1990). Maintenance, Reverse Engineering and Design Recovery. Special Issue of IEEE Software, Vol. 7, No. 1. Jan. 1990.

Software (1994). Systematic Reuse. Special Issue of IEEE Software, Vol. 11, No. 5. Sept. 1994.

Teorey, T. J., Yang, D., Fry J. P. (1986). A Logical Design Methodology for Relational Databases using the Extended ER Model. ACM Computing Surveys, Vol. 18.

\section{BIOGRAPHY}

Univ.-Ass. Hubert Hasenauer received MSc and the $\mathrm{PhD}$ degrees from the University of Bodenkultur, Vienna, Austria, where he currently holds a position as a full time researcher and lecturer. Prior he was a visiting scientist at the College of Forestry and Wildlife Resources at the Virginia Institute of Technology, Blacksburg, VA. His major research interests are inventory design, growth simulation, and computer applications in forestry.

Univ.-Doz. Günther Pernul received MSc and the $\mathrm{PhD}$ degrees from the University of Vienna where he currently holds a senior lecturer position. Shortly he will take over a position as a full professor of information systems at the University of Essen, Germany. His main research interests are information systems analysis, modelling and design, computer security, and database management systems. 